\title{
Descripción de las Diferentes Indicaciones del Cuerpo Adiposo de la Mejilla como Colgajo e Injerto Autólogo en Cirugía Maxilofacial. Revisión Narrativa
}

\author{
Buccal Fat Pad Indications as a Flap and Autologous \\ Graft in Maxillofacial Surgery. Narrative Review
}

Jack Altschiller Mardones ${ }^{1}$; Alfredo Noguera Pantoja ${ }^{1,2}$; Tamara Muñoz Zavala1; Penelhope Pooley Donoso ${ }^{1} \&$ Pedro Solé Ventura ${ }^{1,2}$

\begin{abstract}
ALTSChILleR, M. J.; NOGUERA, P. A.; MUÑOZ, Z. T.; POOLEY, D. P. \& SOLÉ, V. P. Descripción de las diferentes indicaciones del cuerpo adiposo de la mejilla como colgajo e injerto autólogo en cirugía maxilofacial. Revisión narrativa. Int. J. Odontostomat., 12(4):362-367, 2018.

RESUMEN: Uno de los objetivos de la cirugía maxilofacial es devolver anatomía, función y estética a través de distintas técnicas quirúrgicas. Dentro de los tejidos utilizados para esto se encuentra el cuerpo adiposo de la mejilla (CAM). La gran evidencia clínica existente y el conocimiento que se tiene acerca de este tejido ha permitido poder utilizarlo como injerto o colgajo para el tratamiento de una serie de condiciones que afectan al territorio maxilofacial con buenos resultados tanto estéticos como funcionales. Describir las diferentes indicaciones que tiene el CAM como colgajo e injerto autólogo para el tratamiento de las distintas anomalías que afectan al territorio maxilofacial. Se realizó una búsqueda entre abril y junio de 2018 utilizando 3 bases de datos electrónicas: PubMed, EBSCO y Cochrane. Las palabras clave utilizadas fueron buccal fat pad, bichat's fat pad y oral surgery con la selección del término booleano AND y OR. Se seleccionaron aquellos estudios publicados entre los 2014 y 2018, de texto completo. Se seleccionaron 17 artículos de que cumplieron con los criterios de inclusión; 2 revisiones sistemáticas, 8 ensayos clínicos, 6 estudios observacionales y una serie de casos. El uso del CAM como injerto y colgajo para la corrección de anomalías en el territorio maxilofacial es una técnica versátil y predecible. La totalidad de los estudios analizados avalan su uso en dicho campo. Se necesitan más estudios clínicos aleatorizados, con un mayor número de casos, seguimiento a largo plazo y parámetros a evaluar para establecer una conclusión final sobre el uso del CAM en las diversas aplicaciones de la cirugía maxilofacial.
\end{abstract}

PALABRAS CLAVE: cuerpo adiposo de la mejilla, cuerpo adiposo de Bichat, cirugía oral.

\section{INTRODUCCIÓN}

En el territorio maxilofacial cuando no es posible reparar un defecto congénito o adquirido mediante la aposición de los márgenes por medio de suturas, se buscan otras alternativas como la reparación por segunda intención. Para ello se han utilizado distintas técnicas, ya sea a partir del uso de injertos o colgajos. La diferencia entre ambos radica en que si el tejido utilizado para cubrir el defecto se traslada manteniendo su aporte sanguíneo, mediante un puente o pedículo, se denomina colgajo. Por el contrario, si el tejido utilizado se traslada sin mantener el aporte sanguíneo original y por ende depende del aporte vascular de la zona receptora, se denomina injerto (Fernandes,
2014; Volgas et al., 2012; Vuik, 2012). Dentro de los diversos tejidos utilizados se encuentran aquellos provenientes del tejido adiposo, especialmente del cuerpo adiposo de la mejilla (CAM). El injerto de tejido adiposo no es una técnica reciente. Sus primeras aplicaciones se remontan al año 1893, cuando el cirujano alemán Gustav Neuber utilizó por primera vez un injerto adiposo del antebrazo para rellenar una cicatriz facial secundaria a una osteítis tuberosa. El uso de este tejido como injerto se extiende hasta la actualidad, debido a que los avances científicos han permitido considerar al tejido adiposo como un excelente material para restaurar alteraciones de volumen y con-

\footnotetext{
${ }^{1}$ Cirujano Dentista, Universidad de los Andes, Santiago, Chile.

${ }^{2}$ Cirujano Dentista, Cirujano Maxilofacial, Universidad de los Andes, Santiago, Chile.
} 
torno, tanto con fines estéticos como reconstructivos (Pu, 2017; Loukas et al., 2006).

El CAM es una masa lobulada simple, conformada por un cuerpo central y cuatro procesos: bucal, pterigoideo, pterigopalatino y temporal. Se encuentra limitado por el músculo masetero, el tendón del temporal y el pterigoideo medial en la parte posterior, el buccinador y su aponeurosis por medial, el primer plano de los músculos faciales por lateral y el conducto parotídeo junto a su delgada lámina aponeurótica en anterior. El espacio bucal es el compartimento que limita al CAM, junto al conducto parotídeo, glándulas salivales, la arteria facial y vena, arteria bucal, vasos linfáticos, y las ramas de los nervios faciales y mandibulares. Su aporte vascular deriva de las ramas temporales profundas de la arteria maxilar, arteria bucal, ramas faciales transversales de la arteria temporal superficial y de las ramas de la arteria facial (Hwang et al., 2005; Matarasso, 2006). Las funciones del CAM incluyen; Proporcionar acción contraria a la presión negativa de succión por medio de los ligamentos y maximizar la presión necesaria para la alimentación efectiva; como separador de músculos masticatorios y estructuras óseas adyacentes; mejora el movimiento intermuscular y asegura la protección de los haces neurovasculares (Poeschl et al., 2009; Alkan et al., 2003).

En relación a su proceso de cicatrización, clínicamente la superficie de grasa expuesta bucalmente se vuelve blanca amarillenta alrededor de los tres días. Dentro de la primera semana se vuelve gradualmente de color rojo, debido a la formación de tejido de granulación inmaduro. Esto último cambia, debido a que se produce una maduración de tejido de granulación, volviéndose una estructura completamente epitelializada a la 3ra - 4ta semana (Shrime \& Gilbert, 2009; Visscher et al., 2010). Las ventajas de este injerto/colgajo son las siguientes: Ubicación ana- tómica favorable; Técnica quirúrgica rápida y económica; Alta tasa de éxito independiente de la edad, sexo y peso del paciente; Ausencia de cicatrices visibles; Mayor aporte sanguíneo y de células madres, permitiendo aumentar la viabilidad de los tejidos injertados al disminuir el proceso inflamatorio y promover la formación de nuevos vasos sanguíneos; Baja tasa de morbilidad; Puede ser asociados a otros injertos/ colgajos. Las desventajas son las siguientes: Sólo cubre defectos pequeños o medianos debido a su delgadez; Puede reducir la profundidad del vestíbulo dificultando la posterior rehabilitación protésica (Hazarey et al., 2007; Vega et al., 2013). Las indicaciones y contraindicaciones en relación al CAM en el territorio maxilofacial se muestran en la Tabla I. El objetivo general de esta revisión es describir las diferentes indicaciones del CAM como colgajo e injerto autólogo en cirugía maxilofacial.

\section{MATERIAL Y MÉTODO}

Se realizó una búsqueda entre abril y junio del 2018 utilizando 3 bases de datos electrónicas: PubMed, EBSCO y Cochrane. Las palabras clave utilizadas fueron buccal fat pad, bichat's fat pad y oral surgery con la selección del término booleano AND y OR. Se aplicaron filtros a la búsqueda de tiempo e idioma; se aceptaron aquellas publicaciones referentes al tema entre los años 2014 y 2018 y aquellas escritas en inglés o español que se encontraran en texto completo. Dentro de los criterios de selección de los resultados de la búsqueda, se incluyeron sólo aquellos artículos donde se hiciera referencia al CAM como colgajo e injerto autólogo en la región orofacial y en el territorio maxilofacial, ya sea como indicación única o complementario a otros materiales tales como injertos óseos, placas de titanio, entre otros. Además sólo

Tabla I. Indicaciones y contraindicaciones del CAM en el territorio maxilofacial.

\begin{tabular}{ll}
\hline Indicaciones & Contraindicaciones \\
\hline Corrección de Fisura Palatina & $\begin{array}{l}\text { Hipoplasia Malar } \\
\text { Paciente en } \\
\text { Fuente de Células Madres }\end{array}$ \\
Cierre de Comunicación/Fístula Bucosinusal & Mejillas Delgadas \\
Reconstrucción Maxilar & \\
Tratamiento de Osteonecrosis & \\
Tratamiento de Fibrosis Mucosa & \\
Tratamiento de Anquilosis ATM & \\
Elevación de Seno Maxilar & \\
Aumento de Tercio Medio en Cirugía Ortognática & \\
Fractura de Órbita & \\
Síndrome de Frey &
\end{tabular}


ALTSCHILleR, M. J.; NOGUERA, P. A.; MUÑOZ, Z. T.; POOLEY, D. P. \& SOLÉ, V. P. Descripción de las diferentes indicaciones del cuerpo adiposo de la mejilla como colgajo e injerto autólogo en cirugía maxilofacial. revisión narrativa. Int. J. Odontostomat., 12(4):362-367, 2018.

se incluyeron aquellos estudios que fueran realizados en humanos. Se excluyeron aquellas publicaciones que hacían referencia al CAM como tratamiento en zonas anatómicas no referentes a la región maxilofacial $\mathrm{y}$ estudios in vitro en humanos y animales.

Para evaluar la validez de la evidencia recopilada, los artículos seleccionados fueron clasificados según nivel de evidencia y grado de recomendación determinado según los aspectos propuestos por Agència d'Avaluació de Tecnologia Mèdica (AATM) de la Generalitat de Catalunya (Jovell \& Navarro-Rubio, 1995). Los estudios seleccionados fueron criticados desde el punto de vista ético, revisando la presencia de un Comité de Ética institucional, firma de consentimiento informado y descripción de los riesgos potenciales para los pacientes.

\section{RESULTADOS Y DISCUSIÓN}

Al realizar la búsqueda en las bases de datos antes mencionadas, utilizando las palabras clave buccal fat pad, bichat's fat pad y oral surgery con los término booleano AND y OR se obtuvieron 69 resultados. Luego, al aplicar los criterios de inclusión y exclusión, se seleccionaron finalmente 17 publicaciones. De las publicaciones a analizar, se encontraron 2 revisiones sistemáticas, 8 ensayos clínicos, 6 estudios observacionales y 1 estudio de serie de casos (Tablas II-V). EI territorio maxilofacial se ve afectado constantemente por diversas patologías que conducen a la formación de defectos cuya reconstrucción no sólo tiene como objetivo restituir la función sino además la estética. La reparación de los defectos bucales ha sido siempre un reto para el cirujano, no sólo por las limitaciones anatómicas sino también por la naturaleza especializada de los tejidos intraorales. La elección del método de reconstrucción depende de diversos factores tales como la cantidad, localización, tipo y cantidad de tejido que debe ser reemplazado. Para ello diversas técnicas quirúrgicas han sido sugeridas, no obstante, durante las últimas décadas el uso del CAM se ha convertido en una opción ideal para la reconstrucción de estos defectos.
Luego de analizar los 17 artículos seleccionados, se puede establecer que el CAM es un tejido adiposo ideal, pues no sólo proporciona una excelente función sin deteriorar la estética, sino además es realizado mediante una técnica quirúrgica rápida, simple y económica. Presenta una elevada tasa de éxito, la que es independiente de la edad, se y peso del paciente. Los autores establecen en unanimidad que el éxito se debe a que es un tejido con un gran aporte sanguíneo y de células madres, lo que permite aumentar la viabilidad de los tejidos injertados al disminuir el proceso inflamatorio y promover la formación de nuevos vasos sanguíneos. Además, al ser un tejido autólogo es muy poco frecuente que se produzca una respuesta inmune por parte del paciente, llevando al rechazo, infección y extrusión del injerto/colgajo. Si bien dentro de las desventajas son la limitada cantidad de tejido disponible, si se aplica correctamente en casos seleccionados, se traduce en una técnica de éxito seguro. Debido a las múltiples ventajas antes mencionadas, este tejido adiposo se ha convertido en una estructura con una gran variedad de indicaciones. Dentro de las indicaciones encontradas en la literatura, se encuentra la corrección de anomalías congénitas como fisuras palatinas, y la corrección de anomalías adquiridas como comunicaciones y fístulas orosinusales, reconstrucciones maxilares post tratamiento oncológico, tratamiento de osteonecrosis de los maxilares, fibrosis submucosa, anquilosis de articulación temporomandibular, membrana autóloga en cirugía de elevación del seno maxilar,fractura de órbita, síndrome de Frey, fuente de células madres y aumento del tercio medio facial como complemento a cirugía ortognática, entre otras.

En cuanto a la ubicación, este colgajo/injerto ha sido utilizado en múltiples zonas, las cuales no siempre aseguran el éxito de esta técnica. En los 17 estudios analizados la tasa de éxito varía entre $87-100 \%$. Esto se debe a que en los casos con menor tasa de éxito se utiliza este tejido en zonas donde no es recomendado. Es por esto que los autores establecen, a partir de los resultados de sus estudios, que en el maxilar el CAM debe ser utilizado desde el margen alveolar del canino y no más allá de la línea media

Tabla II. Resultado de estudios; Revisiones Sistemáticas.

\begin{tabular}{lcccc}
\hline Revista & Autores/Año & Indicación del CAM & $\begin{array}{c}\text { Nivel de } \\
\text { Evidencia }\end{array}$ & $\begin{array}{c}\text { Fuerza de } \\
\text { Recomendación }\end{array}$ \\
\hline $\begin{array}{l}\text { European Journal } \\
\text { of Oral } \\
\text { Implantology }\end{array}$ & De Biasi et al. (2014) & $\begin{array}{l}\text { Cierre de Comunicación o } \\
\text { Fístula Oroantral }\end{array}$ & I & Adecuada \\
\hline $\begin{array}{l}\text { Cochrane Library } \\
\text { Salian et al. 2016 }\end{array}$ & $\begin{array}{l}\text { Cierre de Comunicación o } \\
\text { Fístula Oroantral }\end{array}$ & I & Adecuada \\
\hline
\end{tabular}


ALTSCHILLER, M. J.; NOGUERA, P. A.; MUÑOZ, Z. T.; POOLEY, D. P. \& SOLÉ, V. P. Descripción de las diferentes indicaciones del cuerpo adiposo de la mejilla como colgajo $e$ injerto autólogo en cirugía maxilofacial. revisión narrativa. Int. J. Odontostomat., 12(4):362-367, 2018.

Tabla III. Resultado de estudios; Ensayos Clínicos.

\begin{tabular}{|c|c|c|c|c|}
\hline Revista & Autor/Año & Indicación del CAM & $\begin{array}{c}\text { Nivel de } \\
\text { Evidencia }\end{array}$ & $\begin{array}{l}\text { Fuerza de } \\
\text { Recomendación }\end{array}$ \\
\hline Oral and Maxillof acial Surgery & Khiabani et al. (2014) & $\begin{array}{c}\text { Cirugía Ortognática (aumento } \\
\text { malar) }\end{array}$ & I & Buena a regular \\
\hline Oral and Maxillof acial Surgery & Bansal et al. (2015) & $\begin{array}{l}\text { Anquilosis de Articulación } \\
\text { Temporomandibular }\end{array}$ & II & Buena a regular \\
\hline Oral and Maxillof acial Surgery & Daif et al. (2016) & $\begin{array}{l}\text { Cierre de Comunicación o } \\
\text { Fístula Oroantral }\end{array}$ & II & Buena a regular \\
\hline Plastic Surgeons & $\begin{array}{l}\text { Hernández-Alfaro et al. } \\
\text { (2015) }\end{array}$ & $\begin{array}{c}\text { Cirugía Ortognática (aumento } \\
\text { malar) }\end{array}$ & III & Buena a regular \\
\hline Human Cell & Kawakami et al. (2017) & Cultivo de Células Madres & III & Regular \\
\hline Oral and Maxillof acial Surgery & $\begin{array}{l}\text { Khojasteh \& Sadeghi } \\
\text { (2016) }\end{array}$ & Cultivo de Células Madres & III & Regular \\
\hline Oral and Maxillof acial Surgery & Lambade et al. (2016) & $\begin{array}{c}\text { Tratamiento de Fibrosis } \\
\text { Mucosa }\end{array}$ & III & Regular \\
\hline $\begin{array}{l}\text { Oral Surgery, Oral Medicine, } \\
\text { Oral Pathology and Oral } \\
\text { Radiology }\end{array}$ & Melville et al. (2016) & Osteonecrosis Medicamentosa & I & Buena a regular \\
\hline
\end{tabular}

Tabla IV. Resultado de estudios. Estudios observacionales.

\begin{tabular}{|c|c|c|c|c|}
\hline Revista & Autor/Año & Indicación del CAM & $\begin{array}{l}\text { Nivel de } \\
\text { Evidencia }\end{array}$ & $\begin{array}{c}\text { Fuerza de } \\
\text { Recomendación }\end{array}$ \\
\hline Oral and Maxillofacial Surgery & Adams et al. (2015) & $\begin{array}{l}\text { Cierre de Comunicación o } \\
\text { Fístula Oroantral }\end{array}$ & V & Regular \\
\hline Oral and Maxillofacial Surgery & Adams et al (2015) & $\begin{array}{l}\text { Cierre de Comunicación o } \\
\text { Fístula Oroantral }\end{array}$ & V & Regular \\
\hline $\begin{array}{l}\text { Clinical and Experimental } \\
\text { Dentistry }\end{array}$ & González et al (2015) & $\begin{array}{l}\text { Cierre de Comunicación o } \\
\text { Fístula Oroantral }\end{array}$ & IV & Buena a regular \\
\hline Craniofacial Surgery & $\begin{array}{l}\text { Chaudhary et al } \\
\text { (2014) }\end{array}$ & $\begin{array}{l}\text { Cierre de Comunicación o } \\
\text { Fístula Oroantral }\end{array}$ & IV & Buena a regular \\
\hline Oral and Maxillofacial Surgery & Rotaru et al. (2015) & $\begin{array}{l}\text { Tratamiento Osteonecrosis } \\
\text { Medicamentosa }\end{array}$ & V & Regular \\
\hline $\begin{array}{l}\text { Maxillofacial Plastic and } \\
\text { Reconstructive Surgery }\end{array}$ & Kim et al. (2017) & Reconstrucción de Defectos & IV & Buena a regular \\
\hline
\end{tabular}

Tabla V. Resultados de estudios; Serie de Casos.

\begin{tabular}{lcccc}
\hline Revista & Autor/Año & \multicolumn{2}{c}{ Indicación del CAM } & Nivel de Evidencia \\
\hline Contemporary Clinical Dentistry & $\begin{array}{c}\text { Agarwal et al. } \\
(2014)\end{array}$ & $\begin{array}{l}\text { Tratamiento } \\
\text { Gingival }\end{array}$ & Recesión & IX \\
\hline
\end{tabular}

palatina, hasta la zona de la tuberosidad en posterior. No obstante, si los defectos son mayores y se encuentran cerca de la línea media, se han realizado colgajos de CAM bilaterales. Mientras que, en mandíbula se recomienda su utilización de forma unilateral y hasta la zona retromolar. Si bien, la ubicación del defecto juega un rol fundamental, los autores relatan que el factor crítico para el éxito del CAM es el tamaño; aunque la literatura reporta que defectos de hasta $7 \times 5 \times$ $2 \mathrm{~cm}$ se han rehabilitado con éxito, la mayoría de los autores recomiendan el uso del CAM en defectos no mayores a $5 \times 4 \mathrm{~cm}$.

En cuanto a la técnica, éste puede ser utilizado de forma aislada o en combinación con otros colgajos/ injertos. Autores estiman que el uso de forma aislada del CAM tiene un buen pronóstico, no obstante, la mayor desventaja es la reducción del fondo de vestíbulo que puede generar problemas en aquellos pacientes que necesiten rehabilitación protésica a futuro. Esta limitación puede eliminarse al utilizar el colgajo/injerto de CAM en conjunto con otros tejidos. Además, al utilizarse este colgajo en doble capa mejora aún más el pronóstico. Por otra parte, la mayor desventaja es que es una técnica más sensible y requiere más tiempo. Aunque a modo general la historia establece que las complicaciones más frecuentes del uso del CAM son necrosis parcial del colgajo/injerto, alteración del contorno de las mejillas y asimetría facial, la siguiente revisión narrativa arroja resultados distintos. Se estableció que si bien la tasa de complicación es mínima, las complicaciones más frecuentes son: edema y dolor, reducción del fondo de vestíbulo, fístula residual, trismus y dehiscencia. 
En relación al seguimiento de los estudios, para la mayoría de estos fue de 4 semanas a 3-6 meses, no obstante, una cantidad no menor de estudios se extendían hasta los 48 meses. Esto último se debe a que se trataban de estudios que utilizaban el CAM tras la resección de tumores malignos, por lo que la duración prolongada de seguimiento fue para detectar cualquier signo de recurrencia de las lesiones. En todos los estudios se estimó que el CAM sanó alrededor de las 2 semanas y la epitelización fue completa a las 46 semanas. Es por esto que se necesitan estudios de mayor tiempo de seguimiento para poder responder a todas las interrogantes y establecer el verdadero éxito de este tejido a lo largo del tiempo.

Al evaluar la calidad de la evidencia, el 47,1\% estudios presentaron evidencia buena, el 11,8 \% presentaron evidencia adecuada, 35,3\% regular y el 5,9 $\%$ presentó evidencia inadecuada. La evidencia analizada corresponde en su mayoría a estudios observacionales, no obstante, en aquellas aplicaciones recientes del CAM en cirugía maxilofacial la mayoría corresponden a reportes de casos clínicos. El $87,5 \%$ de los estudios fueron aprobados por un comité de ética, es decir, existe conocimiento de si existió una supervisión de las investigaciones en seres humanos de manera ética en la mayoría de los estudios utilizados en esta revisión narrativa.

De un total de 17 publicaciones seleccionadas para esta revisión de la literatura científica, la totalidad de ellas avalan el uso del CAM como colgajo e injerto en cirugía maxilofacial. No obstante, si bien existe una gran cantidad de estudios de calidad adecuada para las aplicaciones más frecuentes del CAM, se necesitan más estudios clínicos aleatorizados, con un mayor número de casos, seguimiento a largo plazo y parámetros a evaluar para establecer una conclusión final sobre el uso del CAM en las diversas aplicaciones de la cirugía maxilofacial.

ALTSCHILLER, M. J.; NOGUERA, P. A.; MUÑOZ, Z. T.; POOLEY, D. P. \& SOLÉ, V. P. Buccal fat pad indications as a flap and autologous graft in maxillofacial surgery. Narrative review. Int. J. Odontostomat., 12 (4):362-367, 2018.

ABSTRACT: One of the main objectives of maxillofacial surgery is to restore patient normal anatomy, function and esthetic. Among the various tissues used to achieve the above, is the buccal fat pad. Currently, there is sufficient clinical evidence and knowledge regarding this tissue type, to approve its use as graft or flap, in order to treat a number of conditions that affect the maxillofacial area.
Furthermore, it has shown various esthetical as well as functional results. The purpose of this narrative review is to describe different indications of the buccal fat pad, as a flap and autologous graft in treating various maxillofacial abnormalities. A search was conducted between april and june 2018 using 3 electronic data bases: PubMed, EBSCO and Cochrane library. Key words used were buccal fat pad, Bichat's fat pad y oral surgery using Boolean terms AND/ OR. Studies published between 2014 and 2018 were selected. For the study, 17 complete text studies that met the inclusion criteria were selected; two systematic reviews, eight randomized clinical trials, six observational studies and one case series. The use of the buccal fat pad as flap and graft for correcting maxillofacial abnormalities, is a predictable and versatile technique. The total number of studies analyzed, support its use in this surgical field. In conclusion and despite these results however, there is a need for further randomized clinical trials with a greater number of cases, long term follow up, and greater parameter assessment to support the use of the buccal fat pad in maxillofacial surgery.

KEY WORDS: buccal fat pad, bichat's fat pad y oral surgery.

\section{REFERENCIAS BIBLOGRÁFICAS}

Adams, T.; Taub, D. \& Rosen, M. Repair of oroantral communications by use of a combined surgical approach: functional endoscopic surgery and buccal advancement flap/buccal fat pad graft. $J$. Oral Maxillofac. Surg., 73(8):1452-6, 2015.

Agarwal, C.; Gayathri, G. V. \& Mehta, D. S. An innovative technique for root coverage using pedicled buccal fat pad. Contemp. Clin. Dent., 5(3):386-8, 2014.

Alkan, A.; Dolanmaz, D.; Uzun, E. \& Erdem, E. The reconstruction of oral defects with buccal fat pad. Swiss Med. Wkly.,133(3334):465-70, 2003.

Bansal, V.; Bansal, A.; Mowar, A. \& Gupta, S. Ultrasonography for the volumetric analysis of the buccal fat pad as an interposition material for the management of ankylosis of the temporomandibular joint in adolescent patients. Br. J. Oral Maxillofac. Surg., 53(9):820-5, 2015.

Chaudhary, B.; Gong, Z.; Lin, Z.; Abbas, K.; Ling, B. \& Liu, H. Reconstruction of intraoral maxillary defect with buccal fat pad. J. Craniofac. Surg., 25(6):2174-7, 2014.

Daif, E. T. Long-term effectiveness of the pedicled buccal fat pad in the closure of a large oroantral fistula. J. Oral Maxillofac. Surg., 74(9):1718-22, 2016.

De Biasi, M.; Maglione, M. \& Angerame, D. The effectiveness of surgical management of oroantral communications: a systematic review of the literature. Eur. J. Oral Implantol., 7(4):347-57, 2014.

Fernandes, R. Local and Regional Flaps in Head \& Neck Reconstruction. A Practical Approach. Oxford, John Wiley \& Sons, 2014.

Hazarey, V. K.; Erlewad, D. M.; Mundhe, K. A. \& Ughade, S. N. Oral submucous fibrosis: study of 1000 cases from central India. $J$. Oral Pathol. Med., 36(1):12-7, 2007.

Hernández-Alfaro, F.; Valls-Ontañón, A.; Blasco-Palacio, J. C. \& Guijarro-Martínez, R. Malar augmentation with pedicled buccal fat pad in orthognathic surgery: three-dimensional evaluation. Plast. Reconstr. Surg., 136(5):1063-7, 2015. 
ALTSChILLER, M. J.; NOGUeRA, P. A.; MUÑOZ, Z. T.; POOLEY, D. P. \& SOLÉ, V. P. Descripción de las diferentes indicaciones del cuerpo adiposo de la mejilla como colgajo e injerto autólogo en cirugía maxilofacial. revisión narrativa. Int. J. Odontostomat., 12(4):362-367, 2018.

Hwang, K.; Cho, H. J.; Battuvshin, D.; Chung, I. H. \& Hwang, S. H. Interrelated buccal fat pad with facial buccal branches and parotid duct. J. Craniofac. Surg., 16(4):658-60, 2005.

Jovell, A. J. \& Navarro-Rubio, M. D. Evaluación de la evidencia científica. Med. Clin. (Barc.)., 105(19):740-3, 1995.

Kawakami, M.; Ishikawa, H.; Tanaka, A. \& Mataga, I. Induction and differentiation of adipose-derived stem cells from human buccal fat pads into salivary gland cells. Hum. Cell, 29(3):101-10, 2017.

Khiabani, K.; Keyhan, S. O.; Varedi, P.; Hemmat, S.; Razmdideh, R. \& Hoseini, E. Buccal fat pad lifting: an alternative open technique for malar augmentation. J. Oral Maxillofac. Surg., 72(2):403.e115, 2014.

Khojasteh, A. \& Sadeghi, N. Application of buccal fat pad-derived stem cells in combination with autogenous iliac bone graft in the treatment of maxillomandibular atrophy: a preliminary human study. Int. J. Oral Maxillofac. Surg., 45(7):864-71, 2016.

Kim, M. K.; Han, W. \& Kim, S. G. The use of the buccal fat pad flap for oral reconstruction. Maxillofac. Plast. Reconstr. Surg., 39(1):5, 2017.

Lambade, P.; Dawane, P. \& Thorat, A. Efficacy of buccal fat pad in the surgical management of oral submucous fibrosis: a prospective study. Oral Maxillofac. Surg., 20(2):167-70, 2016.

Loukas, M.; Kapos, T.; Louis, R. G. Jr.; Wartman, C.; Jones, A. \& Hallner, B. Gross anatomical, CT and MRI analyses of the buccal fat pad with special emphasis on volumetric variations. Surg. Radiol. Anat., 28(3):254-60, 2006.

Matarasso, A. Managing the buccal fat pad. Aesthet. Surg. J., 26(3):330-6, 2006.

Melville, J. C.; Tursun, R.; Shum, J. W.; Young, S.; Hanna, I. A. \& Marx, R. E. A technique for the treatment of oral-antral fistulas resulting from medication-related osteonecrosis of the maxilla: the combined buccal fat pad flap and radical sinusotomy. Oral Surg. Oral Med. Oral Pathol. Oral Radiol., 122(3):287-91, 2016.

Poeschl, P. W.; Baumann, A.; Russmueller, G.; Poeschl, E.; Klug, C. \& Ewers, R. Closure of oroantral communications with Bichat's buccal fat pad. J. Oral Maxillofac. Surg., 67(7):1460-6, 2009.

Rotaru, H.; Kim, M. K.; Kim, S. G. \& Park, Y. W. Pedicled buccal fat pad flap as a reliable surgical strategy for the treatment of medication-related osteonecrosis of the jaw. J. Oral Maxillofac. Surg., 73(3):437-42, 2015.

Shrime, M. G. \& Gilbert, R. W. Reconstruction of the midface and maxilla. Facial Plast. Surg. Clin. North Am.,17(2):211-23, 2009.

Vega, L. G. González-García, R. \& Louis, P. J. Reconstruction of acquired temporomandibular joint defects. Oral Maxillofac. Surg. Clin. North Am., 25(2):251-69, 2013.

Visscher, S. H.; van Minnen, B. \& Bos, R. R. Closure of oroantral communications: a review of the literature. J. Oral Maxillofac. Surg., 68(6):1384-91, 2010.

\author{
Dirección para correspondencia: \\ Pedro Solé Ventura \\ Monseñor Álvaro del Portillo 12455, Las Condes \\ Universidad de los Andes \\ Santiago \\ CHILE
}

E-mail: drpedrosole@gmail.com

Recibido : 25-06-2018

Aceptado: 17-09-2018 